\title{
Rigidity of polyhedral surfaces, III
}

\author{
FENG LUO
}

\begin{abstract}
This paper investigates several global rigidity issues for polyhedral surfaces including inversive distance circle packings. Inversive distance circle packings are polyhedral surfaces introduced by P Bowers and K Stephenson [4] as a generalization of Andreev and Thurston's circle packing. They conjectured that inversive distance circle packings are rigid. We prove this conjecture using recent work of $\mathrm{R}$ Guo [9] on the variational principle associated to the inversive distance circle packing. We also show that each polyhedral metric on a triangulated surface is determined by various discrete curvatures that we introduced in [11], verifying a conjecture in [11]. As a consequence, we show that the discrete Laplacian operator determines a spherical polyhedral metric.
\end{abstract}

14E20, 54C40; 46E25, 20C20

To Dennis Sullivan on the occasion of his seventieth birthday

\section{Introduction}

\subsection{Background}

This is a continuation of the study of polyhedral surfaces in $[11 ; 13]$. The paper focuses on inversive distance circle packings introduced by Bowers and Stephenson and several other rigidity issues. Using recent work of Guo [9], we prove a conjecture of Bowers and Stephenson that inversive distance circle packings are rigid. Namely, a Euclidean inversive distance circle packing on a compact surface is determined up to scaling by its discrete curvature. This generalizes an earlier result of Andreev [1] and Thurston [16] on the rigidity of circle packing with acute intersection angles.

In [11], using 2-dimensional Schlaefli formulas, we introduced two families of discrete curvatures for polyhedral surfaces and conjectured that each of one these discrete curvatures determines the polyhedral metric (up to scaling in the Euclidean case). We verify this conjecture in the paper. One consequence is that the cotangent discrete Laplacian operator determines spherical polyhedral metrics. We also reprove the similar theorem for Euclidean polyhedral surfaces obtained by Gu, Guo, Luo and Zeng [8]. 
The main theorems in the paper are proved using variational principles and are based on the work of $[9 ; 11]$. The main idea of the paper comes from readings of Bobenko, Pinkall and Springborn [2], Colin de Verdière [17] and Rivin [14]. We follow the approach pioneered by Colin de Verdière [17].

\subsection{Polyhedral rigidity through discrete curvatures}

Recall that a triangulated surface is a surface obtained by gluing pairs of edges of a collection of disjoint triangles by homeomorphisms. A Euclidean (or spherical or hyperbolic) polyhedral surface is a triangulated surface with a metric, called a polyhedral metric, so that each triangle in the triangulation is isometric to a Euclidean (or spherical or hyperbolic) triangle. To be more precise, let $\mathbf{E}^{2}, \mathbf{S}^{2}$ and $\mathbf{H}^{2}$ be the Euclidean, the spherical and the hyperbolic 2-dimensional geometries. Suppose $(S, T)$ is a closed triangulated surface so that $T$ is the triangulation, and $E$ and $V$ are the sets of all edges and vertices. A $K^{2}\left(=\mathbf{E}^{2}, \mathbf{S}^{2}\right.$ or $\left.\mathbf{H}^{2}\right)$ polyhedral metric on $(S, T)$ is a map $l: E \rightarrow \mathbf{R}$ so that whenever $e_{i}, e_{j}, e_{k}$ are three edges of a triangle in $T$, then

$$
l\left(e_{i}\right)+l\left(e_{j}\right)>l\left(e_{k}\right),
$$

and if $K^{2}=\mathbf{S}^{2}$, in addition to the inequalities above, one requires

$$
l\left(e_{i}\right)+l\left(e_{j}\right)+l\left(e_{k}\right)<2 \pi .
$$

Given $l: E \rightarrow \mathbf{R}$ satisfying the inequalities above, there is a metric on the surface $S$, called a polyhedral metric, so that the restriction of the metric to each triangle is isometric to a triangle in $K^{2}$ geometry and the length of each edge $e$ in the metric is $l(e)$. We also call $l: E \rightarrow \mathbf{R}$ the edge length function. For instance, the boundary of a generic convex polytope in the 3-dimensional space $\mathbf{E}^{3}, \mathbf{S}^{3}$ or $\mathbf{H}^{3}$ of constant curvature 0,1 or -1 is a polyhedral surface. The discrete curvature $k$ of a polyhedral surface is a function $k: V \rightarrow \mathbf{R}$ so that $k(v)=2 \pi-\sum_{i=1}^{m} a_{i}$ where the $a_{i}$ 's are the angles at the vertex $v$. See Figure 1 .



(a)



(b)

Figure 1 
Since the discrete curvature is built from inner angles of triangles, we consider inner angles of triangles as the basic unit of measurement of curvature. Using inner angles, we introduced three families of curvature-like quantities in [11]. The relationships between the polyhedral metrics and curvatures are the focus of this paper.

Definition 1.1 [11] Let $h \in \mathbf{R}$. Given a $K^{2}$ polyhedral metric on $(S, T)$ where $K^{2}=\mathbf{E}^{2}, \mathbf{S}^{2}$ or $\mathbf{H}^{2}$, the $\phi_{h}$ curvature of a polyhedral metric is the function $\phi_{h}: E \rightarrow \mathbf{R}$ sending an edge $e$ to

$$
\phi_{h}(e)=\int_{\pi / 2}^{a} \sin ^{h}(t) d t+\int_{\pi / 2}^{a^{\prime}} \sin ^{h}(t) d t,
$$

where $a, a^{\prime}$ are the inner angles facing the edge $e$. See Figure 1 .

The $\psi_{h}$ curvature of the metric is the function $\psi_{h}: E \rightarrow \mathbf{R}$ sending an edge $e$ to

$$
\psi_{h}(e)=\int_{0}^{(b+c-a) / 2} \cos ^{h}(t) d t+\int_{0}^{\left(b^{\prime}+c^{\prime}-a^{\prime}\right) / 2} \cos ^{h}(t) d t,
$$

where $b, b^{\prime}, c, c^{\prime}$ are inner angles adjacent to the edge $e$ and $a, a^{\prime}$ are the angles facing the edge $e$. See Figure 1 .

The curvatures $\phi_{0}$ and $\psi_{0}$ were first introduced by Rivin [14] and Leibon [10] respectively. If the surface $S=\mathbf{S}^{2}$, then these curvatures are essentially the dihedral angles of the associated 3-dimensional hyperbolic polyhedra at edges. The curvature $\phi_{-2}(e)=-\cot (a)-\cot \left(a^{\prime}\right)$ is the discrete (cotangent) Laplacian operator on a polyhedral surface derived from the finite element approximation of the smooth Beltrami Laplacian on Riemannian manifolds.

One of the remarkable theorems proved by Rivin [14] is that a Euclidean polyhedral metric on a triangulated surface is determined up to scaling by its $\phi_{0}$ discrete curvature. In particular, he proved that an ideal convex hyperbolic polyhedron is determined up to isometry by its dihedral angles.

Theorem 1.2 Let $(S, T)$ be a closed triangulated connected surface. Then for any $h \in \mathbf{R}$,

(1) a Euclidean polyhedral metric on $(S, T)$ is determined up to isometry and scaling by its $\phi_{h}$ curvature,

(2) a spherical polyhedral metric on $(S, T)$ is determined up to isometry by its $\phi_{h}$ curvature,

(3) a hyperbolic polyhedral surface is determined up to isometry by its $\psi_{h}$ curvature. 
We remark that Theorem 1.2(1) for $h=0$ was aforementioned Rivin's theorem. However, our proof of Rivin's theorem is different from that in [14] and we use the variational principle established by Cohen, Kenyon and Propp [6]. Theorem 1.2(3) for $h=0$ was first proved by Leibon [10]. Theorem 1.2(2) for $h=0$ was proved in [12] and Theorem 1.2(2) and (3) for $h \leq-1$ or $h \geq 0$ was proved in [11].

Taking $h=-2$ in Theorem 1.2, we obtain:

Corollary 1.3 (1) [8] A connected Euclidean polyhedral surface is determined up to scaling by its discrete Laplacian operator.

(2) A spherical polyhedral surface is determined by its discrete Laplacian operator.

Note that for a Euclidean polyhedral surface, $\phi_{h}=\psi_{h}$. There remain two questions on whether $\phi_{h}$ curvature determines a hyperbolic polyhedral surface or whether $\psi_{h}$ curvature determines a spherical polyhedral surface. It seems the results may still be true in these cases.

\subsection{Rigidity of inversive distance circle packings}

Inversive distance circle packings are polyhedral metrics on a triangulated surface introduced by Bowers and Stephenson [4]. An expansion of the discussion of [4] is in Bowers and Hurdal [3]. See also Stephenson [15]. They are generalizations of Andreev and Thurston's circle packings. Unlike the case of Andreev and Thurston where adjacent circles are intersecting, Bowers and Stephenson allow adjacent circles to be disjoint and measure their relative positions by the inversive distance. As observed in [4], this relaxation of intersection condition is very useful for practical applications of circle packing to many fields, including medical imaging and computer graphics. Based on extensive numerical evidences, they conjectured the rigidity and convergence of inversive distance circle packings in [4]. Our result shows that Bowers and Stephenson's rigidity conjecture holds. The proof is based on recent work of Guo [9] which established a variational principle for inversive distance circle packings. A very nice geometric interpretation of the variational principle was given by Glickenstein [7].

We begin by briefly recalling the inversive distance in Euclidean, hyperbolic and spherical geometries. See Bowers and Hurdal [3] for a more detailed discussion. Let $K^{2}$ be $\mathbf{E}^{2}, \mathbf{H}^{2}$ or $\mathbf{S}^{2}$. Given two circles $C_{1}, C_{2}$ in $K^{2}$ centered at $v_{1}, v_{2}$ of radii $r_{1}$ and $r_{2}$ so that $v_{1}, v_{2}$ are of distance $l$ apart, the inversive distance $I=I\left(C_{1}, C_{2}\right)$ between the circles is given by

$$
I=\frac{l^{2}-r_{1}^{2}-r_{2}^{2}}{2 r_{1} r_{2}}
$$


in the Euclidean plane,

$$
I=\frac{\cosh (l)-\cosh \left(r_{1}\right) \cosh \left(r_{2}\right)}{\sinh \left(r_{1}\right) \sinh \left(r_{2}\right)}
$$

in the hyperbolic plane and

$$
I=\frac{\cos (l)-\cos \left(r_{1}\right) \cos \left(r_{2}\right)}{\sin \left(r_{1}\right) \sin \left(r_{2}\right)}
$$

in the 2-sphere. See [9] for more details on (1-4) and (1-5). If one considers $\mathbf{E}^{2}, \mathbf{H}^{2}$ and $\mathbf{S}^{2}$ as appeared in the infinity of the hyperbolic 3 -space $\mathbf{H}^{3}$, then $C_{1}$ and $C_{2}$ are the boundaries of two totally geodesic hyperplanes $D_{1}$ and $D_{2}$. The inversive distance $I$ is essentially the hyperbolic distance (or the intersection angle) between $D_{1}$ and $D_{2}$. In particular, for the Euclidean plane $\mathbf{E}^{2}$, the inversive distance $I\left(C_{1}, C_{2}\right)$ is invariant under the inversion and hence the name.

Bowers and Stephenson's construction of an inversive distance circle packing with prescribed inversive distance on a triangulated surface $(S, T)$ is as follows. Fix once and for all a vector $I \in[-1, \infty)^{E}$, called the inversive distance.

In the Euclidean case, for any $r \in \mathbf{R}_{>0}^{V}$, called the radius vector, define the edge length function $l \in \mathbf{R}_{>0}^{E}$ by the formula

$$
l(e)=\sqrt{r(v)^{2}+r(u)^{2}+2 r(v) r(u) I(e)},
$$

where the end points of the edge $e$ is $\{u, v\}$. If for any three edges $e_{i}, e_{j}, e_{k}$ of a triangle in $T$, the triangle inequality

$$
l\left(e_{i}\right)+l\left(e_{j}\right)>l\left(e_{k}\right)
$$

holds, then the length function $l: E \rightarrow \mathbf{R}$ sending $e$ to $l(e)$ defines a Euclidean polyhedral metric on $(S, T)$ called the inversive distance circle packing with inversive distance $I(e)$ at edge $e$. Note that if $I(e) \in[0,1]$ for all $e$, the polyhedral metric is the circle packing investigated by Andreev and Thurston where the intersection angle between two circles at the end points of an edge is $\arccos (I(e))$.

In the hyperbolic geometry, one uses

$$
l(e)=\cosh ^{-1}(\cosh (r(v)) \cosh (r(u))+I(e) \sinh (r(v)) \sinh (r(u)))
$$

as the length of an edge. If (1-7) holds, then the lengths define a hyperbolic inversive distance circle packing with inversive distances $I$ on $(S, T)$. The spherical inversive distance circle packing is defined similarly with additional condition on the $l(e)$ 's that for each triangle with edges $e_{i}, e_{j}, e_{k}$,

$$
l\left(e_{i}\right)+l\left(e_{j}\right)+l\left(e_{k}\right)<2 \pi .
$$


The geometric meaning of these polyhedral metrics is the following. In each metric, if one draws a circle of radius $r(v)$ at each vertex $v$, then inversive distance of two circles at the end points of an edge $e$ is the given number $I(e)$.

Our result which solves Bowers and Stephenson's rigidity conjecture is the following.

Theorem 1.4 Given a closed triangulated connected surface $(S, T)$ with the set of edges $E$ and $I \in \mathbf{R}_{\geq 0}^{E}$ considered as the inversive distance,

(1) a hyperbolic inversive distance circle packing metric on $(S, T)$ of inversive distance $I$ is determined by its discrete curvature $k: V \rightarrow \mathbf{R}$.

(2) a Euclidean inversive distance circle packing metric on $(S, T)$ of inversive distance $I$ is determined by its discrete curvature $k: V \rightarrow \mathbf{R}$ up to scaling.

Note that for $I \in[0,1]^{E}$, the above result was Andreev and Thurston's rigidity for circle packing with intersection angles between $[0, \pi / 2]$. It seems the similar result may be true for $I \in[-1, \infty)^{E}$.

\subsection{Plan of paper}

The paper is organized as follows. In Section 2, we prove an extension lemma for angles of triangles. We also establish a criterion for extending a locally convex function to convex function. In Section 3, we prove Theorem 1.4. Theorem 1.2 is proved in Section 4.

The following notation and conventions will be used in the paper. We use $\mathbf{R}, \mathbf{R}_{>0}$, $\mathbf{R}_{\geq 0}, \mathbf{R}_{<0}$ to denote the sets of all real numbers, positive real numbers, nonnegative real numbers, and negative real numbers respectively. If $X$ is a set, $\mathbf{R}^{X}=\{f: X \rightarrow \mathbf{R}\}$ is the vector space of all functions on $X$. If $A$ is a subspace of a topological space $X$, then the closure of $A$ in $X$ is denoted by $\bar{A}$.

Acknowledgements We thank Ren Guo and the referee for comments and careful reading of the manuscript.

The work is supported in part by a NSF grant number CCF-0830572.

\section{Convex extension of locally convex functions}

The main idea of extension in this section comes from reading of Bobenko, Pinkall and Springborn [2] in which extension of a nonconvex action function to convex one was first carried out in the field. We show that similar extensions can be established in a very general setting. 


\subsection{Continuous extension by constants}

Definition 2.1 Suppose $A$ is a subspace of a topological space $X$ and $f: A \rightarrow Y$ is continuous. If there exists a continuous function $F: X \rightarrow Y$ so that $\left.F\right|_{A}=f$ and $F$ is a constant function on each connected component of $X-A$, then we say $f$ can be extended continuously by constant functions to $X$.

Note that if each connected component of $X-A$ intersects the closure of $A$, then the extension function $F$ is uniquely determined by $f$.

The key observation of the paper is the following simple lemma.

Lemma 2.2 Let $l_{1}, l_{2}, l_{3}$ and $\theta_{1}, \theta_{2}, \theta_{3}$ be the edge lengths and inner angles of a triangle $\Delta$ in $\mathbf{E}^{2}$, or $\mathbf{H}^{2}$, or $\mathbf{S}^{2}$ so that the $l_{i}$-th edge is opposite to the angle $\theta_{i}$. Consider $\theta_{i}=\theta_{i}(l)$ as a function of $l=\left(l_{1}, l_{2}, l_{3}\right)$.

(1) If $\Delta$ is Euclidean or hyperbolic, the angle function $\theta_{i}$ defined on

$$
\Omega=\left\{\left(l_{1}, l_{2}, l_{3}\right) \in \mathbf{R}^{3} \mid l_{1}+l_{2}>l_{3}, l_{2}+l_{3}>l_{1}, l_{3}+l_{1}>l_{2}\right\}
$$

can be extended continuously by constant functions to a function $\tilde{\theta}_{i}$ on $\mathbf{R}_{>0}^{3}$.

(2) If $\Delta$ is spherical, the angle function $\theta_{i}$ defined on

$$
\Omega=\left\{\left(l_{1}, l_{2}, l_{3}\right) \in \mathbf{R}^{3} \mid l_{1}+l_{2}>l_{3}, l_{2}+l_{3}>l_{1}, l_{3}+l_{1}>l_{2}, l_{1}+l_{2}+l_{3}<2 \pi\right\}
$$

can be extended continuously by constant functions to a function $\tilde{\theta}_{i}$ on $(0, \pi)^{3}$.

We call the set $\Omega$ in the lemma the natural domain of the length vectors.

Proof In case (1), the extension function $\tilde{\theta}_{i}$ of $\theta_{i}$ is given by $\tilde{\theta}_{i}=\pi$ when $l_{i} \geq l_{j}+l_{k}$, and $\tilde{\theta}_{i}=0$ when $l_{j} \geq l_{i}+l_{k}$. To verify the continuity of $\tilde{\theta}_{i}$ on $\mathbf{R}_{>0}^{3}$, we use the cosine law. Given a point $L=\left(L_{1}, L_{2}, L_{3}\right)$ in the boundary $\bar{\Omega}-\Omega$ of $\Omega$ inside $\mathbf{R}_{>0}^{3}$, we may assume without loss of generality that $L_{1}=L_{2}+L_{3}$. Continuity of $\tilde{\theta}_{i}$ follows from

$$
\lim _{l \rightarrow L} \theta_{1}(l)=\pi, \quad \lim _{l \rightarrow L} \theta_{j}(l)=0, \quad j=2,3 .
$$

Indeed, the cosine law says, in the case of $\Delta \subset \mathbf{E}^{2}$, that

$$
\cos \left(\theta_{i}\right)=\frac{l_{j}^{2}+l_{k}^{2}-l_{i}^{2}}{2 l_{j} l_{k}} .
$$


One sees easily that when $l$ tends to $L$, then the right-hand-side of (2-1) tends to 1 if $i=2,3$ and -1 if $i=1$. This verifies the continuity in the Euclidean case. In the hyperbolic case, the cosine law says

$$
\cos \left(\theta_{i}\right)=\frac{\cosh \left(l_{j}\right) \cosh \left(l_{k}\right)-\cosh \left(l_{i}\right)}{\sinh \left(l_{j}\right) \sinh \left(l_{k}\right)} .
$$

Thus one sees that as $l$ tends to $L=\left(L_{1}, L_{2}, L_{3}\right)$ with $L_{j}>0$ and $L_{1}=L_{2}+L_{3}$, the right-hand-side of (2-2) tends to 1 if $i=2,3$ and to -1 if $i=1$. Thus $\tilde{\theta}_{i}$ is continuous.

To see (2), recall that the cosine law for spherical triangle says

$$
\cos \left(\theta_{i}\right)=\frac{\cos \left(l_{i}\right)-\cos \left(l_{j}\right) \cos \left(l_{k}\right)}{\sin \left(l_{j}\right) \sin \left(l_{k}\right)} .
$$

If $l$ tends to $L$ where $L_{1}=L_{2}+L_{3}$ with $L_{i} \in(0, \pi)$, then $\lim _{l \rightarrow L} \cos \left(\theta_{1}\right)=-1$ and $\lim _{l \rightarrow L} \cos \left(\theta_{i}\right)=1$ when $i=2,3$. On the other hand, if $L_{1}+L_{2}+L_{3}=2 \pi$ for $L_{i} \in(0, \pi)$, then the cosine law implies that $\lim _{l \rightarrow L} \cos \left(\theta_{i}\right)=-1$ for all $i$, ie, all inner angles are $\pi$ in this case. Thus by setting the extended function $\tilde{\theta}_{i}$ in $(0, \pi)^{3}$ to be $\tilde{\theta}_{i}(l)=\pi$ if $l_{i} \geq l_{j}+l_{k}, \tilde{\theta}_{i}(l)=0$ if $l_{j} \geq l_{i}+l_{k}$, and $\tilde{\theta}_{i}(l)=\pi$ if $l_{i}+l_{j}+l_{k} \geq \pi$, $(\{i, j, k\}=\{1,2,3\})$, we see that $\tilde{\theta}_{i}$ is continuous.

\subsection{Continuous extension of 1-forms and of locally convex functions}

We establish some simple facts on extending closed 1-forms and locally convex functions to convex functions in this subsection.

Definition 2.3 A differential 1-form $w=\sum_{i=1}^{n} a_{i}(x) d x_{i}$ in an open set $U \subset \mathbf{R}^{n}$ is said to be continuous if each $a_{i}(x)$ is a continuous function on $U$. A continuous 1 -form $w$ is called closed if $\int_{\partial \tau} w=0$ for each Euclidean triangle $\tau$ in $U$.

By the standard approximation theory, if $w$ is closed and $\gamma$ is a piecewise $C^{1}$-smooth null homologous loop in $U$, then $\int_{\gamma} w=0$. If $U$ is simply connected, then the integral $F(x)=\int_{a}^{x} w$ is well defined, independent of the choice of piecewise smooth paths in $U$ from $a$ to $x$. The function $F(x)$ is $C^{1}$-smooth so that $\partial F(x) / \partial x_{i}=a_{i}(x)$.

Proposition 2.4 Suppose $X$ is an open set in $\mathbf{R}^{n}$ and $A \subset X$ is an open subset bounded by a smooth (n-1)-dimensional submanifold in $X$. If $w=\sum_{i=1}^{n} a_{i}(x) d x_{i}$ is a continuous $1-$ form on $X$ so that $\left.w\right|_{A}$ and $\left.w\right|_{X-\bar{A}}$ are closed where $\bar{A}$ is the closure of $A$ in $X$, then $w$ is closed in $X$. 
Proof Since closedness is a local property and is invariant under smooth change of coordinates in $X$, we may assume that $X=\mathbf{R}^{n}$ and $A=\left\{\left(x_{1}, \ldots, x_{n}\right) \in \mathbf{R}^{n} \mid x_{n}>0\right\}$. Take a Euclidean triangle $\tau \subset X$. To verify $\int_{\partial \tau} w=0$, we may assume that $\tau$ is not in $\bar{A}$ or $\overline{X-A}$ since otherwise $\int_{\partial \tau} w=0$ follows from the assumption and the standard approximation theorem. In the remaining case, $\tau$ intersects both $\bar{A}$ and $\overline{X-A}$. The plane $x_{n}=0$ cuts the triangle $\tau$ into a triangle $\gamma_{1}$ and a quadrilateral or a triangle $\gamma_{2}$ so that $\gamma_{1}$ and $\gamma_{2}$ are in the closure of $\bar{A}$ and $\overline{X-A}$. We can express, in the singular chain level, $\partial \tau=\partial \gamma_{1}+\partial \gamma_{2}$. By definition, $\int_{\partial \gamma_{i}} w=0$ for each $i$. Thus $\int_{\partial \tau} w=\int_{\partial \gamma_{1}} w+\int_{\partial \gamma_{2}} w=0$.

A real analytic codimension-1 submanifold $Y$ in an open set $X$ in $\mathbf{R}^{n}$ is a smooth submanifold so that locally $Y$ is defined by $k(x)=0$ for a nonconstant real analytic function $k$. Note that if $L$ is a (compact) line segment in $X$, then either $L \subset Y$ or $L \cap Y$ is a finite set. This is due to the fact that a nonconstant real analytic function on an open interval has isolated zeros.

Recall that a function $f$ defined on a convex set $X \subset \mathbf{R}^{n}$ is called convex if for all $p, q \in X$ and all $t \in[0,1], t f(p)+(1-t) f(q) \geq f(t p+(1-t) q)$. It is called strictly convex if for all $p \neq q$ in $X$ and all $t \in(0,1), t f(p)+(1-t) f(q)>f(t p+(1-t) q)$. A function $f$ defined in an open set $U \subset \mathbf{R}^{n}$ is said to be locally convex (or locally strictly convex) if it is convex (or strictly convex) in a convex neighborhood of each point.

Proposition 2.5 Suppose $X \subset \mathbf{R}^{n}$ is an open convex set and $A \subset X$ is an open subset of $X$ bounded by a codimension-1 real analytic submanifold in $X$. If $w=$ $\sum_{i=1}^{n} a_{i}(x) d x_{i}$ is a continuous closed $1-$ form on $X$ so that $F(x)=\int_{a}^{x} w$ is locally convex in $A$ and in $X-\bar{A}$, then $F(x)$ is convex in $X$.

Proof Since $X$ is simply connected, the function $F$ is well defined. To verify convexity, take $p, q \in X$ and consider $f(t)=F(t p+(1-t) q)$ for $t \in[0,1]$. It suffices to show that $f(t)$ is convex in $t$. Since $F$ is $C^{1}$-smooth, $f$ is $C^{1}$-smooth. Let $\partial A=\bar{A}-A$ and $L$ be the line segment from $p$ to $q$. Since $\partial A$ is real analytic, either $L$ intersects $\partial A$ in a finite set of points, or $L$ is in $\partial A$. In the first case, let $0=t_{0}<t_{1}<\cdots<t_{n}=1$ be the partition of $[0,1]$ so that the line segment $t p+(1-t) q$ for $t \in\left(t_{i}, t_{i+1}\right)$ is either in $A$ or in $X-\bar{A}$. By definition, $f(t)$ is convex in $\left[t_{i}, t_{i+1}\right]$, ie, $f^{\prime}(t)$ is increasing in $\left[t_{i}, t_{i+1}\right]$ for $i=0, \ldots, n-1$. Since $f^{\prime}(t)$ is continuous in $[0,1]$, this implies that $f^{\prime}(t)$ is increasing in $[0,1]$, ie, $f(t)$ is convex in $[0,1]$. In the second case that $L \subset \partial A$, we take two sequences of points $p_{m}$ and $q_{m}$ converging to $p$ and $q$ respectively in $X$ so that $p_{m}, q_{m}$ are not in $\partial A$. Then by the case just proved, the functions $f_{m}(t)=F\left(t p_{m}+(1-t) q_{m}\right)$ are convex in $t$. Furthermore, $f_{m}$ converges to $f$. Thus $f$ is convex. 
Corollary 2.6 Suppose $X \subset \mathbf{R}^{n}$ is an open convex set and $A \subset X$ is an open subset of $X$ bounded by a real analytic codimension-1 submanifold in $X$. If $w=$ $\sum_{i=1}^{n} a_{i}(x) d x_{i}$ is a continuous closed $1-$ form on $A$ so that $F(x)=\int_{a}^{x} w$ is locally convex on $A$ and each $a_{i}$ can be extended continuously to $X$ by constant functions to a function $\widetilde{a_{i}}$ on $X$, then $\widetilde{F}(x)=\int_{a}^{x} \sum_{i=1}^{n} \widetilde{a_{i}} d x_{i}$ is a $C^{1}$-smooth convex function on $X$ extending $F$.

We remark that the real analytic assumption in the Proposition 2.5 and Corollary 2.6 can be relaxed to $C^{1}$-smooth. Indeed, one uses the transversality theorem to show that for generic choice of points $p, q$ in $X$, the line segment $L$ from $p$ to $q$ intersects $\partial A$ in a finite set. The same argument shows that $\left.F\right|_{L}$ is convex. By taking the limit, one proves the same result.

\section{A proof of Bowers and Stephenson's Rigidity Conjecture}

We begin by recalling Guo's work on a variational principle associated to inversive distance circle packings and then prove Theorem 1.4. We will work in Euclidean and hyperbolic geometries only.

\subsection{Guo's variational principle for inversive distance circle packing}

Suppose $\Delta$ is a triangle with vertices $v_{1}, v_{2}, v_{3}$ and edges $e_{i j}=v_{i} v_{j}, i \neq j$. Fix once and for all an inversive distance $I_{i j} \in[0, \infty)$ at each edge $e_{i j}$. Then for each assignment of positive number $r_{i}$ at $v_{i}$ for $i=1,2,3$, let

$$
l_{k}=\sqrt{r_{i}^{2}+r_{j}^{2}+2 r_{i} r_{j} I_{i j}}
$$

for Euclidean geometry and

$$
l_{k}=\cosh ^{-1}\left(\cosh \left(r_{i}\right) \cosh \left(r_{j}\right)+I_{i j} \sinh \left(r_{i}\right) \sinh \left(r_{j}\right)\right)
$$

for hyperbolic geometry where $\{i, j, k\}=\{1,2,3\}$.

Let $\Omega=\left\{\left(x_{1}, x_{2}, x_{3}\right) \in \mathbf{R}_{>0}^{3} \mid x_{i}+x_{j}>x_{k},\{i, j, k\}=\{1,2,3\}\right\}$. If $\left(l_{1}, l_{2}, l_{3}\right)$ is in $\Omega$, then we construct a Euclidean triangle $\Delta$ with length $l_{k}$ of $e_{i j}$ given by (3-1) and a hyperbolic triangle, still denoted by $\Delta$, with length $l_{k}$ of $e_{i j}$ given by (3-2). Suppose the angle of the triangle at $v_{i}$ is $\theta_{i}$ and consider $\theta_{i}$ as a function of $\left(r_{1}, r_{2}, r_{3}\right)$. 
Theorem 3.1 (Guo [9]) Fix any $\left(I_{12}, I_{23}, I_{31}\right) \in[0, \infty)^{3}$.

(1) For Euclidean triangles, let $u_{i}=\ln r_{i}$, then the differential 1-form $w=$ $\sum_{i=1}^{3} \theta_{i} d u_{i}$ is closed in the open subset of $\mathbf{R}^{3}$ where it is defined. The integral $F(u)=\int_{u_{0}}^{u} w$ is a well defined locally concave function in $u=\left(u_{1}, u_{2}, u_{3}\right)$ and is strictly locally concave in $u_{1}+u_{2}+u_{3}=0$. Furthermore, if $c \in \mathbf{R}$ and $F(u)$ is defined, then $F(u+(c, c, c))=F(u)$.

(2) For hyperbolic triangles, let $u_{i}=\ln \left(\tanh \left(r_{i} / 2\right)\right)$, then the differential 1-form $w=\sum_{i=1}^{3} \theta_{i} d u_{i}$ is closed in the open subset of $\mathbf{R}_{<0}^{3}$ where it is defined. Furthermore, the integral $F(u)=\int_{u_{0}}^{u} w$ is a well defined strictly locally concave function in $u=\left(u_{1}, u_{2}, u_{3}\right)$.

Guo also proved [9] that the open sets where the 1-forms $w$ are defined in Theorem 3.1 are connected and simply connected. Theorem 3.1 is a generalization of an earlier result obtained by Chow and Luo [5]. Guo proved a local and infinitesimal rigidity theorem for inversive distance circle packing using Theorem 3.1. It says that a Euclidean inversive distance circle packing is locally determined, up to scaling, by the discrete curvature of the underlying polyhedral surface. He also proved the local and infinitesimal rigidity for hyperbolic inversive distance circle packings.

\subsection{Concave extension of Guo's action functional}

Our main observation is that Guo's differential 1 -forms $w=\sum_{i=1}^{3} \theta_{i} d u_{i}$ can be extended to a closed 1-form on $\mathbf{R}^{3}$ in the Euclidean case and on $\mathbf{R}_{<0}^{3}$ in the hyperbolic case so that the integrations of the extended 1 -forms are still concave.

Proposition 3.2 Let $w$ be the 1 -forms defined in Theorem 3.1.

(a) In the case of Euclidean triangles, the 1-form $w$ can be extended to a continuous closed 1-form $\tilde{w}$ on $\mathbf{R}^{3}$ so that the integration $\widetilde{F}(u)=\int_{u_{0}}^{u} \widetilde{w}$ is a $C^{1}$-smooth concave function.

(b) In the case of hyperbolic triangles, the 1-form $w$ can be extended to a continuous closed 1-form $\widetilde{w}$ on $\mathbf{R}_{<0}^{3}$ so that the integration $\widetilde{F}(u)=\int_{u_{0}}^{u} \tilde{w}$ is a $C^{1}$-smooth concave function.

We begin by focusing the 1 -forms in its radius coordinate $r=\left(r_{1}, r_{2}, r_{3}\right) \in \mathbf{R}_{>0}^{3}$. In this case, the 1 -forms are given by $w=\sum_{i=1}^{3} \theta_{i}\left(d r_{i} / r_{i}\right)$ and $w=\sum_{i=1}^{3} \theta_{i}\left(d r_{i} / \sinh \left(r_{i}\right)\right)$. The 1 -form $w$ is defined on the open set $U$ of $\mathbf{R}_{>0}^{3}$ where

$$
U=\left\{\left(r_{1}, r_{2}, r_{3}\right) \in \mathbf{R}_{>0}^{3} \mid l_{i}+l_{j}>l_{k},\{i, j, k\}=\{1,2,3\}\right\},
$$


where $l_{i}=l_{i}\left(r_{1}, r_{2}, r_{3}\right)$ is defined on $\mathbf{R}_{>0}^{3}$. (Note that for hyperbolic and Euclidean geometries, the sets $U$ are different due to (3-1) and (3-2)). The extension of the 1form $w$ is the natural one. Namely, we replace $\theta_{i}$ in $w$ by $\tilde{\theta}_{i}$ appeared in Lemma 2.2. Thus the extended $1-$ form is $\widetilde{w}=\sum_{i=1}^{3} \tilde{\theta}_{i}\left(d r_{i} / r_{i}\right)$ or $\widetilde{w}=\sum_{i=1}^{3} \tilde{\theta}_{i}\left(d r_{i} / \sinh \left(r_{i}\right)\right)$.

It remains to show that $\tilde{w}$ is continuous and closed in $\mathbf{R}_{>0}^{3}$ so that its pull back to the $u$-coordinate has a concave integration. To this end, we prove:

Lemma 3.3 Let $\bar{U}$ be the closure of $U$ in $\mathbf{R}_{>0}^{3}$.

(1) $\theta_{i}$ is a constant function on each connected component of $\bar{U}-U$.

(2) For each connected component $V$ of $\mathbf{R}_{>0}^{3}-U$, the intersection $V \cap \bar{U}$ is a connected component of $\bar{U}-U$.

Proof By (3-3), the boundary $\partial U=\bar{U}-U$ is given by $\bigcup_{i=1}^{3} \partial_{i} U$ where $\partial_{i} U=$ $\left\{\left(r_{1}, r_{2}, r_{3}\right) \in \mathbf{R}_{>0}^{3} \mid l_{i}=l_{j}+l_{k},\{j, k\}=\{1,2,3\}-\{i\}\right\}$. Furthermore, $\mathbf{R}_{>0}^{3}-U=$ $\bigcup_{i=1}^{3} V_{i}$ where $V_{i}=\left\{\left(r_{1}, r_{2}, r_{3}\right) \in \mathbf{R}_{>0}^{3} \mid l_{i} \geq l_{j}+l_{k},\{j, k\}=\{1,2,3\}-\{i\}\right\}$.

First, we note that if $I_{i j} \leq 1$, then $\partial_{k} U=\varnothing$ and $V_{k}=\varnothing$. Indeed, if $I_{i j} \leq 1$, then by (3-1) and (3-2),

$$
l_{k} \leq r_{i}+r_{j}
$$

But due to $I_{a b} \geq 0,(3-1)$ and (3-2), $r_{j}<l_{i}$ and $r_{i}<l_{j}$. Therefore, $l_{k}<l_{i}+l_{j}$. This implies that $\partial_{k} U=\varnothing$ and $V_{k}=\varnothing$.

Next $\partial_{i} U \cap \partial_{j} U=\varnothing$ and $V_{i} \cap V_{j}=\varnothing$ for $i \neq j$. Indeed, if $r \in \partial_{i} U \cap \partial_{j} U$ or $r \in V_{i} \cap V_{j}$, then $l_{i} \geq l_{j}+l_{k}$ and $l_{j} \geq l_{i}+l_{k}$. Thus $l_{k}=0$. But $l_{k}>r_{i}>0$.

We claim that if $I_{i j}>1$, then both $V_{k}$ and $\partial_{k} U$ are nonempty and connected. Assume the claim, then the lemma follows. Indeed, since $l_{s}>0$ for all indices $s$, it follows, by Lemma 2.2, that $\theta_{i}$ is either 0 or $\pi$ in $\partial_{s} U$, ie, (1) holds. Next, $V_{s}$ 's are the connected components of $\mathbf{R}_{>0}^{3}-U$ so that $V_{s} \cap \bar{U}=\partial_{s} U$. Thus (2) holds.

To see the claim, it suffices to show that there is a smooth function $f\left(r_{i}, r_{j}\right)$ defined on $\mathbf{R}_{>0}^{3}$ so that its graph is $\partial_{k} U$ and $V_{k}=\left\{\left(r_{1}, r_{2}, r_{3}\right) \in \mathbf{R}_{>0}^{3} \mid 0<r_{k} \leq f\left(r_{i}, r_{j}\right)\right\}$.

To this end, consider the equation

$$
l_{k}=l_{i}+l_{j}
$$

and let the right-hand-side of (3-4) be $g\left(r_{k}, r_{i}, r_{j}\right)$. We will deal with the Euclidean and hyperbolic geometry separately. 
Case 1 (Euclidean triangles) In this case, the function $g\left(r_{k}, r_{i}, r_{j}\right)$ is given by

$$
g\left(r_{k}, r_{i}, r_{j}\right)=\sqrt{r_{k}^{2}+r_{j}^{2}+2 I_{k j} r_{k} r_{j}}+\sqrt{r_{i}^{2}+r_{k}^{2}+2 I_{i k} r_{i} r_{k}}
$$

Evidently, for a fixed $\left(r_{i}, r_{j}\right) \in \mathbf{R}_{>0}^{2}, g\left(r_{k}, r_{i}, r_{j}\right)$ is a strictly increasing function of $r_{k} \in \mathbf{R}_{>0}$ so that

$$
g\left(0, r_{i}, r_{j}\right)=r_{i}+r_{j}<\sqrt{r_{i}^{2}+r_{j}^{2}+2 I_{i j} r_{i} r_{j}}
$$

(due to $I_{i j}>1$ ) and $\lim _{r_{k} \rightarrow \infty} g\left(r_{k}, r_{i}, r_{k}\right)=\infty$. By the intermediate value theorem, there exists a unique positive number $f\left(r_{i}, r_{j}\right)$ so that

$$
g\left(f\left(r_{i}, r_{j}\right), r_{i}, r_{j}\right)=\sqrt{r_{i}^{2}+r_{j}^{2}+2 r_{i} r_{j} I_{i j}}=l_{k} .
$$

The smoothness of $f\left(r_{i}, r_{j}\right)$ follows from the implicit function theorem applied to (3-4). Indeed,

$$
\frac{\partial g}{\partial r_{k}}=\frac{r_{k}+2 I_{k j} r_{j}}{l_{i}}+\frac{r_{k}+2 I_{i k} r_{i}}{l_{j}}>0 .
$$

Thus, $f\left(r_{i}, r_{j}\right)$ is smooth.

This shows $\partial_{k} U$ is the graph of the smooth function $f$ defined on $\mathbf{R}_{>0}^{2}$, ie,

$$
\partial_{k} U=\left\{\left(r_{1}, r_{2}, r_{3}\right) \in \mathbf{R}_{>0}^{3} \mid r_{k}=f\left(r_{i}, r_{j}\right)\right\} .
$$

Thus it is connected. Now $g\left(r_{k}, r_{i}, r_{j}\right)$ is an increasing function of $r_{k}$, so $V_{k}=$ $\left\{r \in R_{>0}^{3} \mid 0<r_{k} \leq f\left(r_{i}, r_{j}\right),\{i, j\}=\{1,2,3\}-\{k\}\right\}$. Thus $V_{k}$ is connected.

Case 2 (Hyperbolic triangles) By the same argument as in Case 1, it suffices to show the same properties established in Case 1 hold for $g\left(r_{k}, r_{i}, r_{j}\right)$ given by

$$
\begin{aligned}
\cosh ^{-1}\left(\cosh \left(r_{i}\right) \cosh \left(r_{k}\right)\right. & \left.+I_{i k} \sinh \left(r_{i}\right) \sinh \left(r_{k}\right)\right) \\
& +\cosh ^{-1}\left(\cosh \left(r_{k}\right) \cosh \left(r_{j}\right)+I_{k j} \sinh \left(r_{k}\right) \sinh \left(r_{j}\right)\right) .
\end{aligned}
$$

Fix $\left(r_{i}, r_{j}\right) \in \mathbf{R}_{>0}^{2}$. Then the function $g\left(r_{k}, r_{i}, r_{j}\right)$ is clearly strictly increasing in $r_{k} \in \mathbf{R}_{>0}$ so that $\lim _{r_{k} \rightarrow \infty} g\left(r_{k}, r_{i}, r_{j}\right)=\infty$ and due to $I_{i j}>1$,

$$
\begin{aligned}
g\left(0, r_{i}, r_{j}\right) & =r_{i}+r_{j} \\
& =\cosh ^{-1}\left(\cosh \left(r_{i}+r_{j}\right)\right) \\
& =\cosh ^{-1}\left(\cosh \left(r_{i}\right) \cosh \left(r_{j}\right)+\sinh \left(r_{i}\right) \sinh \left(r_{j}\right)\right) \\
& <\cosh ^{-1}\left(\cosh \left(r_{i}\right) \cosh \left(r_{j}\right)+I_{i j} \sinh \left(r_{i}\right) \sinh \left(r_{j}\right)\right)=l_{k} .
\end{aligned}
$$


By the intermediate value theorem, there exists a unique positive number $f\left(r_{i}, r_{j}\right)$ so that $g\left(f\left(r_{i}, r_{j}\right), r_{i}, r_{j}\right)=l_{k}$. The smoothness of $f\left(r_{i}, r_{j}\right)$ follows from the implicit function theorem that

$$
\begin{aligned}
\frac{\partial g}{\partial r_{k}} & =\frac{\cosh \left(r_{i}\right) \sinh \left(r_{k}\right)+I_{i k} \sinh \left(r_{i}\right) \cosh \left(r_{k}\right)}{\sqrt{\left(\cosh \left(r_{i}\right) \cosh \left(r_{k}\right)+I_{i k} \sinh \left(r_{i}\right) \sinh \left(r_{k}\right)\right)^{2}-1}} \\
& +\frac{\cosh \left(r_{j}\right) \sinh \left(r_{k}\right)+I_{j k} \sinh \left(r_{j}\right) \cosh \left(r_{k}\right)}{\sqrt{\left(\cosh \left(r_{j}\right) \cosh \left(r_{k}\right)+I_{j k} \sinh \left(r_{j}\right) \sinh \left(r_{k}\right)\right)^{2}-1}} \\
& >0 .
\end{aligned}
$$

By the same argument as in Case 1, we see that $\partial_{k} U$, being the graph of the smooth function $f$, is connected and $V_{k}$, being the region below the positive function $f$ over $\mathbf{R}_{>0}^{2}$, is also connected.

Now back to the proof of Proposition 3.2, for part (1), consider the real analytic diffeomorphism $u=u(r): \mathbf{R}_{>0}^{3} \rightarrow \mathbf{R}^{3}$ where $u_{i}=\ln r_{i}$. The differential 1-form $w=\sum_{i=1}^{3} \theta_{i}\left(d r_{i} / r_{i}\right)$ pulls back (via $r=u^{-1}(r)$ ) to $w=\sum_{i=1}^{3} \theta_{i} d u_{i}$ as appeared in Theorem 3.1. By Lemma 3.3, the extension $\widetilde{w}=\sum_{i=1}^{3} \widetilde{\theta}_{i} d u_{i}$ is obtained from $w$ by extending each coefficient $\theta_{i}$ by constant functions on $\mathbf{R}^{3}-u^{-1}(U)$. Thus, by Corollary 2.6, the function $\widetilde{F}(u)=\int_{0}^{u} \tilde{w}$ is a $C^{1}$-smooth concave function in $u \in \mathbf{R}^{3}$ so that

$$
\partial \widetilde{F} / \partial u_{i}=\tilde{\theta}_{i} .
$$

The same argument also works for part (2) since $u=u(r)$ with $u_{i}=\ln \tanh \left(r_{i}\right)$ is a real analytic diffeomorphism from $\mathbf{R}_{>0}^{3}$ onto $\mathbf{R}_{<0}^{3}$.

\subsection{Proof of Theorem 1.4 for Euclidean inversive distance circle packing}

Suppose $d_{1}, d_{2}$ are two inversive circle packing metrics on $(S, T)$ with the same inversive distance $I \in[0, \infty)^{E}$ and the same discrete curvature $a \in \mathbf{R}^{V}$. We will show that $d_{1}$ and $d_{2}$ differ by a scalar.

We will use the notation that if $i \in V$ and $x \in \mathbf{R}^{V}$, then $x_{i}=x(i)$. Let $T^{(2)}$ be the set of all triangles in $T$. If a triangle $s \in T^{(2)}$ has vertices $i, j, k \in V$, then we denote the triangle by $s=\{i, j, k\}$. For circle packing metrics of radii $r \in \mathbf{R}_{>0}^{V}$ with a given inversive distance $I$, we use $u \in \mathbf{R}^{V}$ to denote their logarithm coordinate where $u_{i}=\ln r_{i}$. Let $p$ and $q$ in $\mathbf{R}^{V}$ be the logarithmic coordinates of $d_{1}$ and $d_{2}$ respectively.

We will show $d_{1}=\lambda d_{2}$ by using the locally concave function $F$ and its concave extension $\widetilde{F}=\int_{0}^{u} \widetilde{w}$ appeared in Proposition 3.2 associated to Theorem 3.1(1). 
Define a $C^{1}$-smooth function $W: \mathbf{R}^{V} \rightarrow \mathbf{R}$ by

$$
W(u)=-\sum_{s \in T^{(2)}, s=\{i, j, k\}} \tilde{F}\left(u_{i}, u_{j}, u_{k}\right)+\sum_{i \in V}\left(2 \pi-a_{i}\right) u_{i} .
$$

The function $W$ is convex since it is a summation of convex functions. Furthermore, by the definition of $W,(3-7)$, and the definition of discrete curvature $\left(a_{i}\right), p$ and $q$ are both critical points of $W$. Since $W$ is convex in $\mathbf{R}^{V}$, it follows that

$$
W(t p+(1-t) q)=W(p)
$$

for all $t \in[0,1]$. Since

$$
W(t p+(1-t) q)=\sum_{s \in T^{(2)}, s=\{i, j, k\}} f_{i j k}(t)+\sum_{i \in E}\left(2 \pi-a_{i}\right)\left(t p_{i}+(1-t) q_{i}\right),
$$

where the function

$$
f_{i j k}(t)=-\widetilde{F}\left(t p_{i}+(1-t) q_{i}, t p_{j}+(1-t) q_{j}, t p_{k}+(1-t) q_{k}\right)
$$

is convex, it follows that $f_{i j k}(t)$ is linear in $t \in[0,1]$ for all triangles $s$ with vertices $i, j, k$. This is due to the simple fact that a summation of a convex function with a strictly convex function is strictly convex. By Theorem 3.1 on the local strictly convexity of $-F\left(u_{1}, u_{2}, u_{3}\right)$ on $u_{1}+u_{2}+u_{3}=0$ and $F(u+(c, c, c))=F(u)$, we see that $p_{i}-q_{i}=p_{j}-q_{j}=p_{k}-q_{k}$ for each triangle with vertices $i, j, k \in V$. (To be more precise, if $\left(p_{i}, p_{j}, p_{k}\right)-\left(q_{i}, q_{j}, q_{k}\right) \neq(c, c, c)$ for any $c$, then by Theorem 3.1 $\left.f_{i j k}\right|_{[0, \epsilon]}$ is strictly convex for small $\epsilon>0$ even though $f_{i j k}$ may not be strictly convex in $[0,1])$. Since the surface is connected and each $f_{i j k}$ is linear, we have that $p-q=(c, c, \ldots, c) \in \mathbf{R}^{V}$. This shows that $d_{1}$ and $d_{2}$ differ by a scalar.

\subsection{Proof of Theorem 1.4 for hyperbolic inversive distance circle packing}

The proof is essentially the same as in Section 3.3 and is simpler. For any $r \in \mathbf{R}_{>0}^{V}$, define $u=u(r) \in \mathbf{R}_{<0}^{V}$ by $\left.u_{i}=\ln \tanh \left(r_{i} / 2\right)\right)$. Define the $u$-coordinate of the circle packing metric to be $u=u(r)$ for a circle packing with radii $r \in \mathbf{R}_{>0}^{V}$.

We use the same notation as in Section 3.3. Suppose $p, q \in \mathbf{R}_{<0}^{V}$ are the $u$-coordinates of the two hyperbolic circle packing metrics having the same hyperbolic inversive distance $I \in \mathbf{R}_{\geq 0}^{E}$ and the same discrete curvature $a=\left(\underset{\widetilde{F}}{a_{i}}\right) \in \mathbf{R}^{V}$. Define the action functional $W$ on $\mathbf{R}_{<0}^{V}$ by the same formula (3-8) where $\widetilde{F}$ is the concave function in Proposition 3.2 associated to Theorem 3.1(2). Then the same proof as in Section 3.3 shows that $p=q$. 


\section{Two-dimensional Schlaefli type action functionals and their extensions}

The following was proved by the author in [11]. The proof is a straight forward calculation.

Theorem 4.1 Suppose $\Delta$ is a triangle in the Euclidean plane $\mathbf{E}^{2}$, or the hyperbolic plane $\mathbf{H}^{2}$, or the 2 -sphere $\mathbf{S}^{2}$ so that its edge lengths are $l_{1}, l_{2}, l_{3}$ and its inner angles are $\theta_{1}, \theta_{2}, \theta_{3}$ where the $l_{i}$-th edge is opposite to the angle $\theta_{i}$. Let $h \in \mathbf{R}$ and let $\Omega$ be the natural domain for length vectors appeared in Lemma 2.2.

(1) For a Euclidean triangle,

$$
w_{h}=\sum_{i=1}^{3} \frac{\int_{\pi / 2}^{\theta_{i}} \sin ^{h}(t) d t}{l_{i}^{h+1}} d l_{i}
$$

is a closed 1 -form on $\Omega$. The integral $\int_{-(h, h, h)}^{u} w_{h}$ is locally convex in variable $u=\left(u_{1}, u_{2}, u_{3}\right)$ where $u_{i}=\ln l_{i}$ for $h=0$ and $u_{i}=-l_{i}^{-h} / h$ for $h \neq 0$. Furthermore, $\int_{-(h, h, h)}^{u} w_{h}$ is locally strictly convex in hypersurface $u_{1}+u_{2}+u_{3}=0$.

(2) For a spherical triangle,

$$
w_{h}=\sum_{i=1}^{3} \frac{\int_{\pi / 2}^{\theta_{i}} \sin ^{h}(t) d t}{\sin ^{h+1}\left(l_{i}\right)} d l_{i}
$$

is a closed 1 -form on $\Omega$. The integral $\int_{0}^{u} w_{h}$ is locally strictly convex in $u=\left(u_{1}, u_{2}, u_{3}\right)$ where $u_{i}=\int_{\pi / 2}^{l_{i}} \sin ^{-h-1}(t) d t$.

(3) For a hyperbolic triangle,

$$
w_{h}=\sum_{i=1}^{3} \frac{\int_{\pi / 2}^{\theta_{i}} \sin ^{h}(t) d t}{\sinh ^{h+1}\left(l_{i}\right)} d l_{i}
$$

is a closed 1-form.

(4) For a hyperbolic triangle,

$$
w_{h}=\sum_{i=1}^{3} \frac{\int_{0}^{\frac{1}{2}\left(\theta_{i}-\theta_{j}-\theta_{k}\right)} \cos ^{h}(t) d t}{\operatorname{coth}^{h+1}\left(l_{i} / 2\right)} d l_{i}
$$

is a closed 1-form. The integral $\int_{0}^{u} w_{h}$ is locally strictly convex in $u=$ $\left(u_{1}, u_{2}, u_{3}\right)$ where $u_{i}=\int_{1}^{l_{i}} \operatorname{coth}^{-h-1}(t / 2) d t$. 
We remark that Theorem 4.1(1) for $h=0$ was first proved by Cohen, Kenyon and Propp [6]. In this case, they showed that $F(u+(c, c, c))=F(u)$. In the case of Theorem 4.1(1) with $h \neq 0$, we have $F(c u)=c^{-h} F(u)$ for any constant $c$. Theorem 4.1(4) for $h=0$ was first proved by Leibon [10]. It is proved in [11] that above are the complete list of all 2-dimensional Schlaefli type identities up to scaling.

Next we provide some ingredients which will be used in the proof of Theorem 1.2.

Recall that the natural domain $\Omega$ of the edge length vectors is given by

$$
\Omega=\left\{\left(l_{1}, l_{2}, l_{3}\right) \in \mathbf{R}_{>0}^{3} \mid l_{i}+l_{j}>l_{k},\{i, j, k\}=\{1,2,3\}\right\}
$$

for Euclidean and hyperbolic triangles and

$$
\Omega=\left\{\left(l_{1}, l_{2}, l_{3}\right) \in \mathbf{R}_{>0}^{3} \mid l_{i}+l_{j}>l_{k}, l_{1}+l_{2}+l_{3}<2 \pi,\{i, j, k\}=\{1,2,3\}\right\} .
$$

Let $J$ be the natural interval for each individual length $l_{i}$, ie, $J=\mathbf{R}_{>0}$ for Euclidean and hyperbolic triangles and $J=(0, \pi)$ for spherical triangles. In each case of Theorem 4.1, there exists a real analytic diffeomorphism $g: J \rightarrow g(J)$ from $J$ onto the open interval $g(J)$ so that $u_{i}=g\left(l_{i}\right)$. To be more precise, $g(t)=\ln t$ in the case of $h=0$ of Theorem 4.1(1), $g(t)=-t^{-h} / h(h \neq 0)$ in the case of $h \neq 0$ in Theorem 4.1(1), $g(t)=\int_{\pi / 2}^{t} \sin ^{-h-1}(x) d x$ in the case (2) of Theorem 4.1, $g(t)=\int_{1}^{t} \sinh ^{-h-1}(x) d x$ in case (3) of Theorem 4.1 and $g(t)=\int_{1}^{t} \operatorname{coth}^{-h-1}(x) d x$ in case (4). The real analytic diffeomorphism $u\left(l_{1}, l_{2}, l_{3}\right)=\left(u_{1}, u_{2}, u_{3}\right)$ where $u_{i}=g\left(l_{i}\right)$ sends $J^{3}$ onto the open cube $g(J)^{3}$ in $\mathbf{R}^{3}$.

By Lemma 2.2, each of the angle function $\theta_{i}(l): \Omega \rightarrow \mathbf{R}$ can be extended continuously by constant functions to a continuous function $\tilde{\theta}_{i}(l): J^{3} \rightarrow \mathbf{R}$. Define a continuous 1 -form $\widetilde{w_{h}}$ on $J^{3}$ by replacing $\theta_{i}$ in the definition of $w_{h}$ in Theorem 4.1 by $\tilde{\theta}_{i}$.

Lemma 4.2 The continuous differential 1 -form $\widetilde{w_{h}}$ is closed in $J^{3}$.

Proof By Proposition 2.4 where we take $X=J^{3}$ and $A=\Omega$, it suffices to show that $\widetilde{w_{h}}$ is closed in each connected component $U$ of $J^{3}-\bar{\Omega}$. By Theorem $\left.4.1 \widetilde{w}\right|_{A}$ is closed, the restriction of $\widetilde{w_{h}}$ to $U$ is of the form $\sum_{i=1}^{3} c_{i} d u_{i}$ where $u_{i}=g\left(l_{i}\right)$ and $c_{i}$ is a constant. Thus $\left.\widetilde{w_{h}}\right|_{U}$ is closed.

Proposition 4.3 The pull back 1-form $\left(u^{-1}\right)^{*}\left(\widetilde{w_{h}}\right)$ on $g(J)^{3}$ is a closed 1-form. Furthermore, if $F(u)=\int^{u} w_{h}$ is locally convex in $u(\Omega)$ (ie, in cases (1), (2), (4) of Theorem 4.1), then $\widetilde{F}(u)=\int^{u}\left(u^{-1}\right)^{*}\left(\widetilde{w_{h}}\right)$ is convex in $u$ in $g(J)^{3}$. 
Note that by the construction, if $u \in u(\Omega)$ and $w_{h}=\sum_{i=1}^{3} \alpha_{i, h}(u) d u_{i}$ (as shown in Theorem 4.1) then

$$
\frac{\partial \tilde{F}(u)}{\partial u_{i}}=\alpha_{i, h}(u) .
$$

Furthermore, by definition, the $\phi_{h}$ and $\psi_{h}$ curvatures are sum of two of $a_{i, h}(u)$ 's.

Proof By Corollary 2.6 where we take $X=g(J)^{3}$ and $A=u(\Omega)$, it suffices to show that $u(\Omega)$ is bounded by a real analytic surface in $X$ and $\widetilde{F}(u)$ is convex in $u(\Omega)$ and in each component of $g(J)^{3}-\overline{u(\Omega)}$.

Since $\Omega$ in $J^{3}$ is bounded by hyperplanes and $u(l)=\left(g\left(l_{1}\right), g\left(l_{2}\right), g\left(l_{3}\right)\right)$ is a real analytic diffeomorphism, it follows that $u(\Omega)$ is bounded by a real analytic surface in $g(J)^{3}$.

By the assumption $\widetilde{F}(u)$ is convex in $u(\Omega)$. If $U$ is a connected component of $g(J)^{3}-\overline{u(\Omega)}$, then $\widetilde{F}(u)$ is linear on $U$ since its partial derivatives are constants on $U$ by the construction. Thus by Corollary 2.6, the result follows.

\section{Proof of Theorem 1.2}

The argument is essentially the same as that in Section 3.3. Recall that $E$ is the set of all edges in the triangulated surface $(S, T)$. If $x \in \mathbf{R}^{E}$ and $i \in E$, we use $x_{i}$ to denote $x(i)$. If $s \in T^{(2)}$ is a triangle with edges $i, j, k \in E$, we denote it by $s=\{i, j, k\}$.

\subsection{Proof of Theorem 1.2(3)}

Suppose two hyperbolic polyhedral metrics on $(S, T)$ have the same $\psi_{h}$ curvature $a=\left(a_{i}\right) \in \mathbf{R}^{E}$. We want to show that these two metrics are the same.

Recall that a polyhedral metric on $(S, T)$ is given by its edge length map $l: E \rightarrow \mathbf{R}_{>0}$. In using the variational principle in Theorem 4.1(4), the natural variable is given by $u: E \rightarrow \mathbf{R}$ where $u(e)=g(l(e))$ with $g(t)=\int_{1}^{t} \operatorname{coth}^{h+1}(s / 2) d s$. We call it the $u$-coordinate of the polyhedral metric $l$ and we will use the $u$-coordinate to set up the variational principle. Let $p, q \in g\left(\mathbf{R}_{>0}\right)^{E}$ be the $u$-coordinates of the two metrics so that their $\psi_{h}$ curvatures are the same $a \in \mathbf{R}^{E}$. Define a $C^{1}$-smooth function $W: g\left(\mathbf{R}_{>0}\right)^{E} \rightarrow \mathbf{R}$ by

$$
W(u)=\sum_{s \in T^{(2)}, s=\{i, j, k\}, i, j, k \in E} \tilde{F}\left(u_{i}, u_{j}, u_{k}\right)-\sum_{i \in E} a_{i} u_{i} .
$$


The function $W$ is convex since it is a summation of convex functions. Furthermore, by the definition of $W,(4-1)$, and the definition of $\psi_{h}$ and $\left(a_{i}\right), p$ and $q$ are critical points of $W$. Since $W$ is convex, we get

$$
W(t p+(1-t) q)=W(p)
$$

for all $t \in[0,1]$. On the other hand,

$$
W(t p+(1-t) q)=\sum_{i, j, k \in E,\{i, j, k\} \in T^{(2)}} f_{i j k}(t)-\sum_{i \in E} a_{i}\left(t p_{i}+(1-t) q_{i}\right),
$$

where the function

$$
f_{i j k}(t)=\tilde{F}\left(t p_{i}+(1-t) q_{i}, t p_{j}+(1-t) q_{j}, t p_{k}+(1-t) q_{k}\right)
$$

is convex, it follows that $f_{i j k}(t)$ is linear in $t \in[0,1]$. By Theorem 4.1, the function $f_{i j k}(t)$ is strictly convex for $t$ near 0,1 unless $\left(p_{i}, p_{j}, p_{k}\right)=\left(q_{i}, q_{j}, q_{k}\right)$. By the linearity of $f_{i j k}$ and connectivity of the surface, it follows $p=q$.

\subsection{Proof of Theorem 1.2(2)}

The proof is exactly the same as above using the extended convex function $\widetilde{F}$ in Proposition 4.3 associated to Theorem 4.1(2).

\subsection{Proof of Theorem 1.2(1)}

The proof is the same as that in Section 5.1 using the similarly defined function $W$. To be more precise, let $g(t)=-t^{-h} / h$ for $h \neq 0$ and $g(t)=\ln t$. By the same set up as in Section 5.1, we conclude that $f_{i j k}(t)$ given by (5-1) is linear in $t$. We claim this implies that the two Euclidean polyhedral metrics $u^{-1}(p)$ and $u^{-1}(q)$ differ by a scalar multiplication. There are two cases to be discussed depending on $h=0$ or $h \neq 0$.

Case $1 h=0$. By Theorem 4.1(1), the function $f_{i j k}(t)$ is strictly convex for $t$ close to 0 or 1 unless $\left(p_{i}, p_{j}, p_{k}\right)-\left(q_{i}, q_{j}, q_{k}\right)=(c, c, c)$ for some $c$. Thus by the connectivity of the surface and linearity of $f_{i j k}$, it follows that the two Euclidean polyhedral metrics $u^{-1}(p)$ and $u^{-1}(q)$ differ by a scalar multiplication.

Case $2 h \neq 0$. By Theorem 4.1(1), the function $f_{i j k}(t)$ is strictly convex for $t$ close to 0 or 1 unless $\left(p_{i}, p_{j}, p_{k}\right)=c\left(q_{i}, q_{j}, q_{k}\right)$ for some $c$. By the same argument, we see that the two metrics differ by a scaling. 


\section{References}

[1] E M Andreev, Convex polyhedra in Lobachevskii spaces, Mat. Sb. (N.S.) 81 (123) (1970) 445-478 MR0259734

[2] A Bobenko, U Pinkall, B Springborn, Discrete conformal maps and ideal hyperbolic polyhedra arXiv:1005.2698

[3] P L Bowers, M K Hurdal, Planar conformal mappings of piecewise flat surfaces, from: "Visualization and mathematics III", (H-C Hege, K Polthier, editors), Math. Vis., Springer, Berlin (2003) 3-34 MR2046999

[4] P L Bowers, K Stephenson, Uniformizing dessins and Bely̌ maps via circle packing, Mem. Amer. Math. Soc. 170, no. 805, Amer. Math. Soc. (2004) MR2053391

[5] B Chow, F Luo, Combinatorial Ricci flows on surfaces, J. Differential Geom. 63 (2003) 97-129 MR2015261

[6] H Cohn, R Kenyon, J Propp, A variational principle for domino tilings, J. Amer. Math. Soc. 14 (2001) 297-346 MR1815214

[7] D Glickenstein, Discrete conformal variations and scalar curvature on piecewise flat two- and three-dimensional manifolds, J. Differential Geom. 87 (2011) 201-237 MR2788656

[8] XD Gu, R Guo, F Luo, W Zeng, Discrete Laplace-Beltrami operator determines discrete Riemannian metric arXiv:1010.4070

[9] R Guo, Local rigidity of inversive distance circle packing, Trans. Amer. Math. Soc. 363 (2011) 4757-4776 MR2806690

[10] G Leibon, Characterizing the Delaunay decompositions of compact hyperbolic surfaces, Geom. Topol. 6 (2002) 361-391 MR1914573

[11] F Luo, Rigidity of polyhedral surfaces, to appear in J. Differential Geom. arXiv: 0612.5714

[12] F Luo, A characterization of spherical polyhedral surfaces, J. Differential Geom. 74 (2006) 407-424 MR2269784

[13] F Luo, On Teichmüller spaces of surfaces with boundary, Duke Math. J. 139 (2007) 463-482 MR2350850

[14] I Rivin, Euclidean structures on simplicial surfaces and hyperbolic volume, Ann. of Math. 139 (1994) 553-580 MR1283870

[15] K Stephenson, Introduction to circle packing: The theory of discrete analytic functions, Cambridge Univ. Press (2005) MR2131318

[16] W P Thurston, The geometry and topology of three-manifolds, Princeton Univ. Math. Dept. Lecture Notes (1979) Available at http://msri.org/publications/books/ gt $3 \mathrm{~m} /$ 
[17] Y Colin de Verdière, Un principe variationnel pour les empilements de cercles, Invent. Math. 104 (1991) 655-669 MR1106755

Department of Mathematics, Rutgers University

New Brunswick NJ 08854, USA

fluo@math.rutgers.edu

Proposed: David Gabai

Received: 17 January 2011

Seconded: Dmitri Burago, Jean-Pierre Otal

Revised: 27 August 2011 